\title{
MINIMAL LENGTH FACTORIZATIONS OF FINITE SIMPLE GROUPS OF LIE TYPE BY UNIPOTENT SYLOW SUBGROUPS
}

\author{
MARTINO GARONZI, DAN LEVY, ATTILA MARÓTI, AND IULIAN I. SIMION
}

\begin{abstract}
We prove that every finite simple group $G$ of Lie type satisfies $G=U U^{-} U U^{-}$where $U$ is a unipotent Sylow subgroup of $G$ and $U^{-}$is its opposite. We also characterize the cases for which $G=U U^{-} U$. These results are best possible in terms of the number of conjugates of $U$ in the above factorizations.
\end{abstract}

\section{INTRODUCTION}

Let $G$ be a finite simple group of Lie type with defining characteristic $p$. We address the problem of finding the minimal number $m$ such that $G$ is equal to the product of $m$ Sylow $p$-subgroups (unipotent Sylows) of $G$. This question has already been considered by several authors before us. Liebeck and Pyber had proved [8, Theorem D] that $G$ is a product of no more than 25 Sylow $p$-subgroups. In [1] it was claimed that the 25 can be replaced by 5 , however no complete proof has been published. A sketch of a proof of this claim for exceptional Lie type groups can be found in a survey by Pyber and Szabó [10, Theorem 15]. Smolensky, Sury and Vavilov [16, Theorem 1] considered the problem of unitriangular factorizations of Chevalley groups over commutative rings of stable rank 1 . When specializing their results to elementary Chevalley groups over finite fields, they get that any non-twisted finite simple group of Lie type is a product of four unipotent Sylows. Later on, these results were extended by Smolensky in [11] to cover some twisted Chevalley groups over finite fields or the field of complex numbers.

Here we give a unified self-contained treatment of the problem of finding minimal length products of unipotent Sylows for all finite simple groups of Lie type, by exploiting their split $B N$-pair structure. Our main result is the following theorem.

Theorem 1 Let $G$ be a simple group of Lie type with defining characteristic $p$. Let $U$ be a Sylow p-subgroup of $G$ and $U^{-}$its opposite. Then $G=\left(U U^{-}\right)^{2}$, and moreover, $G=U U^{-} U$ if and only if $U$ is self-normalizing. In both cases these factorizations are of minimal length.

After the completion of our work, and in parallel to its publication in preprint form [6], Smolensky made available a preprint in which he shows that every Suzuki

Date: September 9, 2015.

2000 Mathematics Subject Classification. 20D20, 20D40, $20 \mathrm{E} 42$.

Key words and phrases. groups with a BN-pair, simple groups of Lie type, products of conjugate subgroups, Sylow subgroups.

M.G. acknowledges the support of MIUR-Italy via PRIN Group theory and applications.

A.M. was supported by an Alexander von Humboldt Fellowship for Experienced Researchers, by MTA Rényi "Lendület" Groups and Graphs Research Group, and by OTKA K84233.

I.S. acknowledges the support of the University of Padova (grants CPDR131579/13 and CPDA125818/12). 
and Ree group is a product of four unipotent Sylow subgroups [12]. Thus, the results in [14], [15], [16], [13], [11] and [12], combine to give a different proof of the four Sylow claim of Theorem 1.

\section{Preliminaries}

The proof of Theorem 1 consists of two main steps: 1. A reduction to the case where the Weyl group is $Z_{2}$ (i.e., to groups $G$ of rank 1), which is carried within the framework of groups with a split $B N$-pair, and some extra assumptions to be detailed in the sequel. 2. A derivation of a general necessary and sufficient criterion for rank 1 groups satisfying an extended set of split $B N$-pair assumptions, that is then verified to hold for the special case of groups with a $\sigma$-setup, using a result from [5].

We would like to point out that although the proof of [16, Theorem 1] also uses a "reduction to rank 1 argument" which is due to Tavgen' [14], we do not know if there is a more direct relation between this approach and ours.

We treat simple groups of Lie type in the setting of groups with a $\sigma$-setup as in [7, Definition 2.2.1]. For this fix a prime $p$, a simple algebraic group $\bar{K}$ defined over $\overline{\mathbb{F}}_{p}$ and a Steinberg endomorphism $\sigma$ of $\bar{K}$, and consider $K$ - the subgroup of $C_{\bar{K}}(\sigma)$ generated by all $p$-elements. All groups $K$ obtained in this way are said to have a $\sigma$-setup given by the pair $(\bar{K}, \sigma)$. The set of all groups possessing a $\sigma$-setup for the prime $p$ is denoted by $\mathcal{L} i e(p)$. Set $\mathcal{L} i e:=\cup \mathcal{L} i e(p)$ where the union is over all primes $p$. We have surjective homomorphisms $K_{u} \rightarrow K \rightarrow K_{a}$ with central kernels [7, Theorem 2.2.6], where the groups $K_{u}, K_{a} \in \mathcal{L} i e(p)$ are called the universal and the adjoint version of $K$, respectively. Any finite simple group of Lie type can be realized as the adjoint version $K_{a}$ of some $K \in \mathcal{L} i e$ [7, Definition 2.2.8] (note that the Tits group ${ }^{2} F_{4}(2)^{\prime}$ is not in $\left.\mathcal{L} i e\right)$. For $G \in \mathcal{L} i e$ we have [3, Chapter 2]:

(i) $G$ is a group with a split $B N$-pair $(B, N)$ and a finite Weyl group $W$, where $B=H \ltimes U$

(ii) $U$ is a Sylow $p$-subgroup of $G$,

(iii) $G$ is generated by its $p$-elements.

\section{Reduction to the case $|W|=2$ for groups With a SPlit $B N$-pair}

For our purposes we will call a triple $(H, U, N)$ a split $B N$-pair for a group $G$ if $(H \ltimes U, N)$ satisfies the axioms of split $B N$-pairs in [3, $\S 2.5]$ with respect to $H$ and $U$. We assume that the Weyl group $W=N / H$ of the $B N$-pair is finite (this certainly holds for finite groups), and so the longest element $w_{0}=n_{0} H$ of $W$ exists and defines subgroups $U^{-}:=U^{n_{o}}$ and $B^{-}:=B^{n_{o}}$. For $w \in W$ we sometimes use $\dot{w}$ to denote an arbitrary choice of an element of $N$ such that $w=\dot{w} H$. We use the notation $U^{-}, X_{i}, U_{i}, X_{-i}, U_{w}$ from $[3, \S 2.5]$ for the $B N$-pair $(B, N)$ and we label with the upper-script ' - ' the corresponding subgroups for $\left(B^{-}, N\right)$, i.e. when $U$ and $B$ are replaced by $U^{-}$and $B^{-}$everywhere: $\left(U^{-}\right)^{-}, X_{i}^{-}, U_{i}^{-}, X_{-i}^{-}, U_{w}^{-}$. Note that $\left(U^{-}\right)^{-}=U, X_{i}^{-}=X_{-i}, X_{-i}^{-}=X_{i}$ and that $X_{i}=U_{s}$ and $X_{-i}=U_{s}^{-}$for some simple reflection $s$. In addition, define $L_{s}:=\left\langle U_{s}, U_{s}^{-}, H\right\rangle$ and $G_{s}:=\left\langle U_{s}, U_{s}^{-}\right\rangle$for any simple reflection $s \in W$. Furthermore, we assume that the root subgroups $X_{\alpha}$, $\alpha \in \Phi(\Phi$ is the set of roots associated with $W$ ) satisfy the commutator relations $[3, \mathrm{p} .61]$. 
Lemma 3.1. Let $G$ be a group with a split $B N$-pair $(H, U, N)$. Suppose that $G$ is a product of $k=2 m+\varepsilon \geq 3$ conjugates of $U$ where $m \geq 1$ is an integer and $\varepsilon \in\{0,1\}$. Then $G=\left(U U^{-}\right)^{m} U^{\varepsilon}$.

Proof. By assumption, $G=U^{x_{1}} U^{x_{2}} \cdots U^{x_{k}}$ for some elements $x_{1}, \ldots, x_{k} \in G$. This is equivalent to $G=U g_{1} U \cdots U g_{k-1} U$ for some $g_{1}, \ldots, g_{k-1} \in G[2, \S 2$ Lemma 1]. By the Bruhat expression of elements (w.r.t. $H$ and $U$ ) we may assume that $g_{i} \in N$ for all $i$. Indeed, by [3, Theorem 2.5.14], for each $1 \leq i \leq k-1$, we have $g_{i}=u_{i} h_{i} \dot{w}_{i} u_{i}^{\prime}$ where $u_{i} \in U, h_{i} \in H, w_{i} \in W$ and $u_{i}^{\prime} \in U_{w} \leq U$. Since the $h_{i}$ lie in $N_{G}(U)$ we have $U g_{1} U \cdots U g_{k-1} U=U h_{1} \dot{w}_{1} U \cdots U h_{k-1} \dot{w}_{k-1} U=$ $U \dot{w}_{1} U \cdots U \dot{w}_{k-1} U\left(h_{1}^{\dot{w}_{1} \cdots \dot{w}_{k-1}} \cdots h_{k-1}\right)$. Thus we have proved that $G$ is a product of $k$ conjugates of $U$ if and only if $G=U g_{1} U \cdots U g_{k-1} U$ for some $g_{1}, \ldots, g_{k-1} \in N$, which is equivalent to $G=U U^{g_{1}^{-1}} U^{g_{2}^{-1}} \cdots U^{g_{k-1}^{-1}}$ (same $g_{i}$ - see proof of $[2, \S 2$ Lemma 1]).

Let $n \in N$ be arbitrary, and let $w=n H$. By [3, Proposition 2.5.12] we have

$$
U=U_{w_{0} w} U_{w}=\left(U \cap U^{n_{0}\left(n_{0} n\right)}\right)\left(U \cap U^{n_{0} n}\right),
$$

which gives

$$
U^{n^{-1}}=\left(U \cap U^{n_{0}\left(n_{0} n\right)}\right)^{n^{-1}}\left(U \cap U^{n_{0} n}\right)^{n^{-1}}=\left(U^{n^{-1}} \cap U\right)\left(U^{n^{-1}} \cap U^{n_{0}}\right) \leq U U^{-} .
$$

However, since $U, U_{w_{0} w}, U_{w}$ are all subgroups, $U=U_{w_{0} w} U_{w}$ implies $U=U_{w} U_{w_{0} w}$, and hence we also get $U^{n^{-1}} \leq U^{-} U$. Therefore

$G=U U^{g_{1}^{-1}} U^{g_{2}^{-1}} \cdots U^{g_{k-1}^{-1}} \subseteq U\left(U U^{-}\right)\left(U^{-} U\right)\left(U U^{-}\right)\left(U^{-} U\right) \cdots=U U^{-} U U^{-} U \cdots$, where we have used $U^{2}=U$ and $\left(U^{-}\right)^{2}=U^{-}$, and the claim follows.

In the following lemma we collect known results about minimal (non-abelian) Levi subgroups which will be used in the sequel. First note that for a fixed split $B N$ pair $(H, U, N)$ we have the (split) $B N$-pair opposite to $(B, N)$ given by $\left(H, U^{-}, N\right)$. Clearly, for any $g \in G,\left(B^{g}, N^{g}\right)$ is a split $B N$-pair, and if $g \in N$ then $B^{g} \cap N=H$ so $B^{g}=H \ltimes U^{g}$. In particular this applies to $g=n_{0}$.

Lemma 3.2. Let $G$ have a split $B N$-pair $(H, U, N)$. Let $w_{0}$ be the longest element of the Weyl group $N / H$ and $s=n_{s} H$ a simple reflection with respect to $(H, U, N)$. Then

(a) $U=U_{s} U_{w_{0} s}=U_{w_{0} s} U_{s}$ and $U^{-}=U_{s}^{-} U_{w_{0} s}^{-}=U_{w_{0} s}^{-} U_{s}^{-}$,

(b) $L_{s} \subseteq N_{G}\left(U_{w_{0} s}\right) \cap N_{G}\left(U_{w_{0} s}^{-}\right)$.

Proof. Any $s=n_{s} H$ in $W$ is simple with respect to $(H, U, N)$ if and only if it is simple with respect to $\left(H, U^{-}, N\right)$. This follows from [3, Propositions 2.2.6 and 2.2.7] and the fact that the positive roots with respect to $\left(H, U^{-}, N\right)$ are the negative roots with respect to $(H, U, N)$. So $I:=\left\{s_{1}, \ldots, s_{l}\right\}$, the set of simple reflections for $(H, U, N)$, is a set of simple reflections for both of these $B N$ pairs and $s=s_{i}$ for some $i \in\{1, \ldots, l\}$. Since $L_{s}$ is the subgroup $X_{i} H \cup X_{i} H n_{i} X_{i}=$ $\left\langle X_{i}, X_{-i}, H\right\rangle\left[3\right.$, Corollary 2.6.2], $X_{i}^{-}=X_{-i}$ and $X_{-i}^{-}=X_{i}$, it follows that $L_{s}=$ $\left\langle X_{i}^{-}, X_{-i}^{-}, H\right\rangle$ is a (minimal) standard Levi subgroup with respect to both $(B, N)$ and $\left(B^{-}, N\right)$.

Now (a) follows from [3, Proposition 2.5.11] and (b) is a particular case of [3, Proposition 2.6.4]. 
The following lemma is [16, Lemma 4].

Lemma 3.3. Let $G$ be a group and let $X \subseteq G$ satisfy $X=X^{-1}$ and $G=\langle X\rangle$. If $\emptyset \neq Y \subseteq G$ is such that $X Y \subseteq Y$ then $Y=G$.

Proposition 3.4. Let $G$ be a group with a split BN-pair such that the conjugates of $U$ in $G$ generate $G$. Let $k \geq 2$ be an integer and assume further that $G_{s}=\left(U_{s} U_{s}^{-}\right)^{k}$ for every simple reflection $s$ then $G=\left(U U^{-}\right)^{k}$.

Proof. Set $X:=\left\{u^{g} \mid u \in U, g \in G\right\}$. Then $X=X^{-1}$ since $U$ is a subgroup of $G$, and $G=\langle X\rangle$ since $G$ is the normal closure of $U$. Set $Y:=\left(U U^{-}\right)^{k}$. By Lemma 3.3 our claim will follow if we show that $X Y \subseteq Y$. Thus it suffices to show that $u^{g} Y \subseteq Y$ for any $u \in U$ and $g \in G$. By [3, Theorem 2.5.14], for any $g \in G$ there exist $u^{\prime} \in U, h \in H, w \in W$ and $u^{\prime \prime} \in U_{w} \leq U$ such that $g=u^{\prime} h n_{w} u^{\prime \prime}$. Hence $u^{g}=u^{u^{\prime} h n_{w} u^{\prime \prime}}=\left(u^{u^{\prime} h}\right)^{n_{w} u^{\prime \prime}}$. But $u^{u^{\prime} h} \in U$, so it is sufficient to prove that $u^{n v} Y \subseteq Y$ for all $u, v \in U$ and $n \in N$. Now we claim that the last statement follows if we prove that $N$ normalizes $Y$. For suppose that $N$ normalizes $Y=\left(U U^{-}\right)^{k}$. We have:

$$
\begin{aligned}
u^{n v}\left(U U^{-}\right)^{k} & =v^{-1} n^{-1} \text { unv }\left(U U^{-}\right)^{k}=v^{-1} n^{-1} \text { un }\left(U U^{-}\right)^{k} \\
& =v^{-1} n^{-1} u\left(U U^{-}\right)^{k} n=v^{-1} n^{-1}\left(U U^{-}\right)^{k} n \\
& =v^{-1}\left(U U^{-}\right)^{k} n^{-1} n=\left(U U^{-}\right)^{k}
\end{aligned}
$$

Thus we prove that $N$ normalizes $\left(U U^{-}\right)^{k}$. Since $H$ clearly normalizes $\left(U U^{-}\right)^{k}$, and $N$ is generated by a set $I$ of representatives for simple reflections together with $H$, it is sufficient to prove that $\left(U U^{-}\right)^{k}$ is normalized by all $n$ in $I$. Fix a simple reflection $s=n H$. By Lemma 3.2.(a), $U U^{-}=U_{s} U_{w_{0} s} U_{w_{0} s}^{-} U_{s}^{-}$. By Lemma 3.2.(b), each of $U_{s}$ and $U_{s}^{-}$commutes with both $U_{w_{0} s}$ and $U_{w_{0} s}^{-}$. This, and the assumption $G_{s}=\left(U_{s} U_{s}^{-}\right)^{k}$, give:

$$
\left(U U^{-}\right)^{k}=\left(U_{s} U_{s}^{-}\right)^{k}\left(U_{w_{0} s} U_{w_{0} s}^{-}\right)^{k}=G_{s}\left(U_{w_{0} s} U_{w_{0} s}^{-}\right)^{k} .
$$

Since $L_{s}=G_{s} H$ we can assume $n \in G_{s}$ and hence $n G_{s}=G_{s} n$. Since $n \in G_{s} \leq$ $L_{s}$, Lemma 3.2.(b) gives $n\left(U_{w_{0} s} U_{w_{0} s}^{-}\right)^{k}=\left(U_{w_{0} s} U_{w_{0} s}^{-}\right)^{k} n$. Combining everything together yields:

$$
\begin{aligned}
n\left(U U^{-}\right)^{k} & =n G_{s}\left(U_{w_{0} s} U_{w_{0} s}^{-}\right)^{k}=G_{s} n\left(U_{w_{0} s} U_{w_{0} s}^{-}\right)^{k} \\
& =G_{s}\left(U_{w_{0} s} U_{w_{0} s}^{-}\right)^{k} n=\left(U U^{-}\right)^{k} n
\end{aligned}
$$

and the proof that $N$ normalizes $\left(U U^{-}\right)^{k}$ is concluded.

Remark 3.5. If $G$ is generated by $U$ and $U^{-}$then the use of Lemma 3.3 in the above proof can be avoided as follows. If $N$ normalizes $\left(U U^{-}\right)^{k}$ then $\left(U U^{-}\right)^{k}$ is stable under conjugation by $n_{0} H$ and so it is equal to $\left(U^{-} U\right)^{k}$. It is easy to see that if this equality holds then $G=\left(U U^{-}\right)^{k}$.

\section{The CASE $|W|=2$}

In this section we prove (Lemma 4.2) a criterion for a group $G$ of rank 1 to satisfy $G=\left(U U^{-}\right)^{2}$. 
Lemma 4.1. Let $G$ be a group with a split $B N$-pair $(H, U, N)$ and a Weyl group $W=\left\{1, s_{1}\right\}$. Set $\left(U^{-}\right)^{*}:=U^{-}-\{1\}$. Fix an arbitrary $n_{1} \in N$ such that $s_{1}=n_{1} H$, and set

$$
\widetilde{H}:=\left\{h \in H \mid \exists u^{-} \in\left(U^{-}\right)^{*}, U u^{-} U=U n_{1} h U\right\} .
$$

Then:

(a) $U\left(U^{-}\right)^{*} U=U n_{1} \widetilde{H} U$.

(b) $U U^{-} U U^{-}=U U^{-}\left(\{1\} \cup n_{1} \widetilde{H} n_{1} \widetilde{H}\right) \cup U n_{1} \widetilde{H}$.

Proof. (a) Since $W=\left\{1, s_{1}\right\}$ we have

$$
G=B \cup B n_{1} B=U H \cup U H n_{1} H U=U H \cup U n_{1} H U,
$$

where the union on the right is disjoint. By [3, Proposition 2.5.5(i)], $B \cap U^{-}=1$. Hence $\left(U^{-}\right)^{*} \subseteq U n_{1} H U$. Thus, for every $u^{-} \in\left(U^{-}\right)^{*}$ there exists $h \in H$ such that $U u^{-} U=U n_{1} h U$. But, by definition, $h \in \widetilde{H}$, so this proves $U\left(U^{-}\right)^{*} U \subseteq U n_{1} \widetilde{H} U$. The reverse inclusion is also clear and hence $U\left(U^{-}\right)^{*} U=U n_{1} \widetilde{H} U$.

(b) Note that since each element of $H$ normalizes both $U$ and $U^{-}$, the set $\widetilde{H}$ commutes with $U$. Also, $w_{0}=s_{1}$ and hence $n_{1} U n_{1}^{-1}=U^{-}$and $n_{1} U^{-} n_{1}^{-1}=U$. Given this and the relation in (a) we get:

$$
\begin{aligned}
U U^{-} U U^{-} & =U\left(U^{-}\right)^{*} U U^{-} \cup U U^{-}=U n_{1} \widetilde{H} U U^{-} \cup U U^{-} \\
& =U U^{-} n_{1} \widetilde{H} U^{-} \cup U U^{-}=U U^{-} U n_{1} \widetilde{H} \cup U U^{-} \\
& =U\left(U^{-}\right)^{*} U n_{1} \widetilde{H} \cup U n_{1} \widetilde{H} \cup U U^{-} \\
& =U n_{1} \widetilde{H} U n_{1} \widetilde{H} \cup U n_{1} \widetilde{H} \cup U U^{-} \\
& =U U^{-} n_{1} \widetilde{H} n_{1} \widetilde{H} \cup U n_{1} \widetilde{H} \cup U U^{-} \\
& =U U^{-}\left(\{1\} \cup n_{1} \widetilde{H} n_{1} \widetilde{H}\right) \cup U n_{1} \widetilde{H} .
\end{aligned}
$$

Lemma 4.2. Let $G$ be a group with a split $B N$-pair $(H, U, N)$ and Weyl group $W=$ $\left\{1, s_{1}\right\}$. Using the notation of Lemma 4.1, the following conditions are equivalent:

(a) $\left(U^{-}\right)^{*} \cap U n_{1} h U \neq \emptyset$ for all $h \in H$. Equivalently $H=\widetilde{H}$.

(b) $U\left(U^{-}\right)^{*} U=U n_{1} H U$.

(c) $G=\left(U U^{-}\right)^{2}$.

Proof. By definition $\widetilde{H} \subseteq H$ and by Lemma 4.1 (a), $U\left(U^{-}\right)^{*} U=U n_{1} \widetilde{H} U$. Hence (a) and (b) are equivalent. To finish the proof observe that

$$
\begin{gathered}
G=B \cup B n_{1} B=\left(B \cup B n_{1} B\right) n_{1}=B n_{1} \cup B n_{1} B n_{1}=B n_{1} \cup B B^{-} \\
=U n_{1} H \cup U U^{-} H,
\end{gathered}
$$

where the union on the r.h.s. is disjoint. Since the sets $\widetilde{H}$ and $n_{1} \widetilde{H} n_{1}$ are both contained in $H$, we have $U n_{1} \widetilde{H} \subseteq U n_{1} H$, and $U U^{-}\left(\{1\} \cup n_{1} \widetilde{H} n_{1} \widetilde{H}\right) \subseteq U U^{-} H$. Since $G=U n_{1} H \cup U U^{-} H$ is a disjoint union, Lemma 4.1 (b) implies that $G=$ $\left(U U^{-}\right)^{2}$ if and only if $U n_{1} \widetilde{H}=U n_{1} H$ and $U U^{-}\left(\{1\} \cup n_{1} \widetilde{H} n_{1} \widetilde{H}\right)=U U^{-} H$. Thus, by Lemma 4.1 (a), we get that (c) implies (b), and it is also clear that (a) implies (c). 


\section{GRoups With A $\sigma$-SETUP}

Any $K \in \mathcal{L}$ ie has a split $B N$-pair $(H, U, N)$, where $U$ is a Sylow $p$-subgroup for the defining characteristic $p$, descending from the algebraic group $\bar{K}[7$, Theorem 2.3.4]. More precisely, if $\bar{T} \subseteq \bar{B}$ is a pair of $\sigma$-stable maximal torus and Borel subgroup of $\bar{K}$ then $B=\bar{B} \cap K$ and $N=N_{\bar{K}}(\bar{T}) \cap K$ form a $B N$-pair for $K$ and if $\bar{U}$ is the unipotent radical of $\bar{K}$, i.e., $\bar{B}=\bar{T} \ltimes \bar{U}$, then $B=H \ltimes U$ where $H=\bar{T} \cap K$ and $U=\bar{U} \cap K$ is a Sylow $p$-subgroup of $K$ (as in [7, Section 3.4]).

Remark 5.1. 1.) Some groups in Lie have split $B N$-pairs for different primes $p$, e.g. $A_{1}(4)=A_{1}(5)[7$, Theorem 2.2.10].

2.) For any simple reflection $s$, if $K \in \mathcal{L} i e(p)$ then $K_{s} \in \mathcal{L} i e(p)$, and if $K$ is universal then so is $K_{s}$ by [7, Theorem 2.6.5.(f)].

3.) Note also that if $\bar{K}$ is universal [7, Theorem 1.10.4] then, by a result of Steinberg [9, Theorem 24.15], $K_{u}=C_{\bar{K}}(\sigma)$ so B, $N, H$ and $U$ are the centralizers of $\sigma$ in $\bar{B}, \bar{N}, \bar{T}$ and $\bar{U}$ respectively.

Lemma 5.2. Let $K_{u} \in \mathcal{L}$ ie $(p)$ be universal of rank 1 and let $U$ be a Sylow $p$ subgroup of $K_{u}$. Then $K_{u}=\left(U U^{-}\right)^{2}$.

Proof. First note that since $K_{u}$ is universal, the corresponding algebraic group $\bar{K}_{u}$ is universal (or simply connected in a different terminology [7, Definition 1.10.5]). By Remark 5.1.3, $K_{u}$ is a finite group of Lie type. The possible types for rank 1 are $A_{1},{ }^{2} A_{2},{ }^{2} B_{2}$ and ${ }^{2} G_{2}$ (see for example [9, Table 23.1]) and the possibilities for $\bar{K}_{u}$ can be read off from [7, Theorem 1.10.7].

Let $p$ be the defining characteristic of $K_{u}$. By [3, $\left.\S 1.19\right]$, we need to consider, for all powers $q$ of $p$, the groups $\mathrm{SL}_{2}(q), \mathrm{SU}_{3}\left(q^{2}\right),{ }^{2} B_{2}\left(q^{2}\right)$ if $p=2$ and $q^{2}=2^{2 n-1}$ for some $n \geq 0$ and ${ }^{2} G_{2}\left(q^{2}\right)$ if $p=3$ and $q^{2}=3^{2 n-1}$ for some $n \geq 0$.

Now $K_{u}$ satisfies the assumptions of Lemma 4.2, so, in particular we use the notation of Lemma 4.2. For $K_{u}=\mathrm{SL}_{2}(q)$ condition (a) of the lemma is easily verified - for the calculation see $[4, \S 6.1]$. For the remaining cases we use $[5$, Proposition 4.1]. By this result, for every $h \in H$ there exists $y \in U$ such that $y n_{1} \in U^{-} h U=n_{1}^{-1} U n_{1} h U$. Multiplying by $n_{1}^{-1}$ on the left, and using $\left(n_{1}^{-1}\right)^{2} \in H$, we obtain $n_{1}^{-1} y n_{1} \in U n_{1}\left(\left(n_{1}^{-1}\right)^{2} h\right) U$. Observe that $1 \notin U n_{1}\left(\left(n_{1}^{-1}\right)^{2} h\right) U$, and hence $n_{1}^{-1} y n_{1} \in\left(U^{-}\right)^{*}$. Moreover, as $h$ varies over $H$, so does $\left(n_{1}^{-1}\right)^{2} h$. Hence, condition (a) of Lemma 4.2 holds for this case, and the claim follows.

Remark 5.3. Note that the groups denoted by $\mathrm{SU}_{n}\left(q^{2}\right)$ in $[3, \S 1.19]$ are denoted by $\mathrm{SU}_{n}(q)$ in $\left[9\right.$, Example 21.2]. Note also that for the groups ${ }^{2} B_{2}\left(2^{2 n-1}\right)$ the universal and the adjoint versions are isomorphic [3, §1.19]. Moreover since the center $Z\left(K_{u}\right)$ lies in $C_{Z\left(\bar{K}_{u}\right)}(\sigma)$ [9, Corollary 24.13] it follows that $Z\left(K_{u}\right)=1$ except if $K_{u}=\mathrm{SL}_{2}(q)$ and $q$ is odd (here $Z\left(K_{u}\right)=Z_{2}$ ) or $K_{u}=\mathrm{SU}_{3}\left(q^{2}\right)$ and 3 divides $q+1$ (here $Z\left(K_{u}\right)=Z_{3}$ ). Excluding these exceptions, $K_{u}$ is isomorphic to its adjoint version, i.e. $K_{u} \cong K_{a}$ and condition (a) of Lemma 4.2 can be checked with the calculation in $[4, \S 13.7]$.

The next lemma is an analogue for the split $B N$-pair setting, of an observation of $[16]$.

Lemma 5.4. If $G$ is a group with a split $B N$-pair $(H, U)$, then $H \cap U U^{-} U=\{1\}$. 
Proof. Let $h \in H \cap U U^{-} U$. Then $h \in u_{1} U^{-} u_{2}$, with $u_{1}, u_{2} \in U$. Equivalently, $u_{1}^{-1} h u_{2}^{-1}=h\left(u_{1}^{-1}\right)^{h} u_{2}^{-1} \in U^{-}$. But $h\left(u_{1}^{-1}\right)^{h} u_{2}^{-1} \in B=H U$, and hence $h\left(u_{1}^{-1}\right)^{h} u_{2}^{-1} \in B \cap U^{-}=\{1\}$ [3, Proposition 2.5.5(i)]. Using $H \cap U=\{1\}$ this gives $h=1$.

Proof of Theorem 1. We will show that $G=\left(U U^{-}\right)^{2}$ for each $G \in \mathcal{L} i e$. As explained in Section 2, this set of groups includes all finite simple groups of Lie type (and some more). Since $G$ satisfies the assumptions of Proposition 3.4, we can assume that $G$ is in $\mathcal{L}$ ie of rank 1 . Moreover, since there is a surjective homomorphism $K_{u} \rightarrow K$ which maps unipotent Sylows onto unipotent Sylows, we can assume that $G$ is universal. A universal $G$ in $\mathcal{L}$ ie of rank 1 satisfies $G=\left(U U^{-}\right)^{2}$ by Lemma 5.2 .

Suppose $H \neq 1$. By Lemma 5.4, $H \cap U U^{-} U=\{1\}$ and so, employing Lemma 3.1, $G$ is a product of at least four unipotent Sylow subgroups, and hence $G=\left(U U^{-}\right)^{2}$ is of minimal length. Suppose now that $H=1$. By [2, Theorem 5], $G=B B^{-} B$, where $B=U H$ and $B=U^{-} H$. Substituting $H=1$ gives $G=U U^{-} U$. Moreover, $H=1$ if and only if $U$ is self-normalizing.

\section{REFERENCES}

[1] L. Babai, N. Nikolov, and L. Pyber. Product growth and mixing in finite groups. In Proceedings of the Nineteenth Annual ACM-SIAM Symposium on Discrete Algorithms, pages 248-257. ACM, New York, 2008.

[2] J. Cannon, M. Garonzi, D. Levy, A. Maróti, and I. Simion. Groups equal to a product of three conjugates subgroups. 2014. To appear in Israel journal of Mathematics.

[3] R. W. Carter. Finite groups of Lie type. Pure and Applied Mathematics (New York). John Wiley \& Sons, Inc., New York, 1985. Conjugacy classes and complex characters, A WileyInterscience Publication.

[4] R. W. Carter. Simple groups of Lie type. Wiley Classics Library. John Wiley \& Sons Inc., New York, 1989. Reprint of the 1972 original, A Wiley-Interscience Publication.

[5] V. Chernousov, E. W. Ellers, and Nikolai G., Gauss decomposition with prescribed semisimple part: short proof. J. Algebra, 229(1):314-332, 2000.

[6] M. Garonzi, D. Levy, A. Maróti, and I. Simion. Minimal length products of unipotent Sylow subgroups in finite simple groups of Lie type. arXiv:1501.05678v1, 2015.

[7] D. Gorenstein, R. Lyons, and R. Solomon. The classification of the finite simple groups. Number 3. Part I. Chapter A, volume 40 of Mathematical Surveys and Monographs. American Mathematical Society, Providence, RI, 1998. Almost simple $K$-groups.

[8] M. W. Liebeck and L. Pyber. Finite linear groups and bounded generation. Duke Math. J., 107(1):159-171, 2001

[9] G. Malle and D. M. Testerman. Linear algebraic groups and finite groups of Lie type, volume 133 of Cambridge Studies in Advanced Mathematics. Cambridge University Press, Cambridge, 2011.

[10] L. Pyber and E. Szabó. Growth in linear groups. In Thin groups and superstrong approximation, volume 61 of Math. Sci. Res. Inst. Publ., pages 253-268. Cambridge Univ. Press, Cambridge, 2014.

[11] A. Smolensky. Unitriangular factorization of twisted Chevalley groups. Internat. J. Algebra Comput., 23(6):1497-1502, 2013.

[12] A. Smolensky. Products of Sylow subgroups in Suzuki and Ree groups. arXiv: $1501.05234 \mathrm{v} 1$,arXiv:1501.05234v2, 2015.

[13] A. Smolensky, B. Sury, and N. Vavilov. Gauss decomposition for Chevalley groups, revisited. Int. J. Group Theory, 1(1):3-16, 2012.

[14] O. I. Tavgen'. Bounded generability of Chevalley groups over rings of $S$-integer algebraic numbers. Izv. Akad. Nauk SSSR Ser. Mat., 54(1):97-122, 221-222, 1990. 
[15] O. I. Tavgen'. Bounded generability of Chevalley groups over rings of $S$-integer algebraic numbers.Proceedings of the International Conference on Algebra Dedicated to the Memory of A. I. Mal'cev, Part 1, vol. CONM/131.1, Contemp. Math, 1992, pp. 409-421.

[16] N. A. Vavilov, A. V. Smolenskiǔ, and B. Sury. Unitriangular factorizations of Chevalley groups. Zap. Nauchn. Sem. S.-Peterburg. Otdel. Mat. Inst. Steklov. (POMI), 388(Voprosy Teorii Predstavlenii Algebr i Grupp. 21):17-47, 309-310, 2011.

(Martino Garonzi) Departamento de Matematica, Universidade de Brasília, Campus Universitário Darcy Ribeiro, Brasília - DF 70910-900, Brasil

E-mail address: mgaronzi@gmail.com

(Dan Levy) The School of Computer Sciences, The Academic College of Tel-AvivYaffo, 2 Rabenu Yeruham St., Tel-Aviv 61083, Israel

E-mail address: danlevy@mta.ac.il

(Attila Maróti) Fachbereich Mathematik, Technische Universität Kaiserslautern, Postfach 3049, 67653 Kaiserslautern, Germany, and Alfréd Rényi Institute of Mathematics, ReÁltanoda utca 13-15, H-1053, Budapest, Hungary

E-mail address: maroti@mathematik.uni-kl.de and maroti.attila@renyi.mta.hu

(Iulian I. Simion) Department of Mathematics, University of Padova, Via Trieste 63, 35121 PAdova, ItALY

E-mail address: iulian.simion@math.unipd.it 